\title{
Characteristic Fingerprint Based on Low Polar Constituents for Discrimination of Wolfiporia extensa according to Geographical Origin Using UV Spectroscopy and Chemometrics Methods
}

\author{
Yan Li, ${ }^{1,2}$ Ji Zhang, ${ }^{1}$ Yanli Zhao, ${ }^{1}$ Zhimin $\mathrm{Li}^{1}{ }^{1}$ Tao Li, ${ }^{3}$ and Yuanzhong Wang ${ }^{1}$ \\ ${ }^{1}$ Institute of Medicinal Plants, Yunnan Academy of Agricultural Sciences, Kunming 650200, China \\ ${ }^{2}$ College of Traditional Chinese Medicine, Yunnan University of Traditional Chinese Medicine, Kunming 650500, China \\ ${ }^{3}$ College of Resources and Environment, Yuxi Normal University, Yuxi 653100, China \\ Correspondence should be addressed to Yuanzhong Wang; boletus@126.com
}

Received 22 October 2014; Accepted 16 November 2014; Published 2 December 2014

Academic Editor: Sibel A. Ozkan

Copyright (C) 2014 Yan Li et al. This is an open access article distributed under the Creative Commons Attribution License, which permits unrestricted use, distribution, and reproduction in any medium, provided the original work is properly cited.

\begin{abstract}
The fungus species Wolfiporia extensa has a long history of medicinal usage and has also been commercially used to formulate nutraceuticals and functional foods in certain Asian countries. In the present study, a practical and promising method has been developed to discriminate the dried sclerotium of $W$. extensa collected from different geographical sites based on UV spectroscopy together with chemometrics methods. Characteristic fingerprint of low polar constituents of sample extracts that originated from chloroform has been obtained in the interval $250-400 \mathrm{~nm}$. Chemometric pattern recognition methods such as partial least squares discriminant analysis (PLS-DA) and hierarchical cluster analysis (HCA) were applied to enhance the authenticity of discrimination of the specimens. The results showed that $W$. extensa samples were well classified according to their geographical origins. The proposed method can fully utilize diversified fingerprint characteristics of sclerotium of $W$. extens $a$ and requires low-cost equipment and short-time analysis in comparison with other techniques. Meanwhile, this simple and efficient method may serve as a basis for the authentication of other medicinal fungi.
\end{abstract}

\section{Introduction}

For millennia, fungi have been appreciated by human beings as edible and medical resources. They are extraordinary species of natural medicines that have long been used around the world [1]. Medicinal fungi, which are used as decoctions and essences, are also normally applied as alternative medicine in China, Korea, Japan, and eastern Russia [2, 3]. Many species of fungi with pharmaceutical values are included in Chinese pharmacopoeia as traditional Chinese medicines (TCMs) [4].

Medicinal fungi showed their special therapeutic effects because of the complexity of their chemical components and different varieties of bioactivities [5]. However, chemical composition and bioefficacy are generally affected by the geographical origins, climatic conditions, environment, and other factors that may lead to somewhat different qualities of medicinal fungi even though they come from the same species $[6,7]$. For example, the content of fatty acid in a very famous medicinal fungus, Ophiocordyceps sinensis, which should grow at least $3800 \mathrm{~m}$ above sea level [8], varied significantly due to difference in geographic origins [9]. When properly evaluating the health benefits of medicine chemical components in laboratory or clinical trials, one should know well where the raw material is from [10]. Therefore, a clear regional identity is imperative to guarantee quality and benefit for the exploitation and utilization of medicinal fungi. It was also the focus of attention for fungus growers.

In recent years, chemical fingerprints have attracted an increasing amount of interest and have been accepted as an available strategy for the identification and quality assessment of medicines by WHO and SFDA [11, 12]. Compared with conventional analytical approaches, fingerprint technique is focused on the holistic characterization of a complex system of a test sample $[13,14]$. Numerous chemical 
fingerprint methods for discrimination and quality evaluation of medicinal fungi have been published including near infrared (NIR) spectroscopy, Fourier transform infrared (FTIR) spectroscopy, high-performance liquid chromatography (HPLC), hydrophilic interaction chromatography (HILIC), gas chromatography-mass spectrometry (GC-MS), and DNA sequence analyses [15-20]. Moreover, these well-established analytical methods exhibited significant advantages for discrimination of different geographical origins when combined with chemometrics which focus on soft modeling for situations that are too complicated for the traditional hard models to work and get useful chemical information from the analytical data maximally [21-24]. However, these methods have a series of important drawbacks. For instance, FT-IR had the weaknesses that it needed the experienced technicians and it was hard to develop a suitable model [25]. For HPLC, the sample pretreatment used to be long and tedious, the standards and calibration are required, and it is a time-consuming, expensive, and destructive technique which is also difficult to implement in an online protocol [26]. Comparatively, UV spectra fingerprints which provide the comprehensive fuzz information of specimens and wildly used in medicine analysis have shown greater potential for discrimination of medicinal fungi [27-29]. Yang et al. used UV spectra fingerprints combined with multivariate analysis to discriminate boletes with different origins and species [30]. This approach is simple and cost-effective and could detect samples rapidly when coupled with chemometric data analysis techniques.

Wolfiporia extensa (Peck) Ginns, one species of wooddecaying fungi in the family Polyporaceae, is a well-known medicinal fungus widely used in China and certain Asian countries $[31,32]$. This species gives large edible sclerotia which is one of the most important crude drugs normally used in the form of the decoctions and in combination with some other herbs in traditional Chinese and Japanese medicine $[4,33,34]$. W. extensa contains two principal groups of chemicals: the triterpene fraction and the polysaccharide fraction [35]. Modern phytochemical and pharmacological researches demonstrated that main active constituents such as triterpenoids and polysaccharides isolated from W. extensa had antioxidant, antitumor, anticancer, anti-inflammatory, nematicidal activities, antihypertonic stress effect, and antihyperglycemic property [36-42]. This species fungus has not only long been utilized to treat a wide variety of diseases, but also recently has attracted the attention of the pharmaceutical industry. Traditionally, it has been used as a diuretic, sedative, and tonic to treat diabetes, edema, nephrosis, acute gastroenteric catarrh, chronic fatigue syndrome, insomnia, diarrhea, nausea, emesis, and dizziness [4, 31, 43]. What is more, it is commercially available and is popularly used in the formulation of nutraceuticals, cosmetics, tea supplements, and functional foods in Asia at present [44].

In this study, a practical and promising method has been developed to discriminate the dried sclerotium of $W$. extensa from different geographical origins based on UV spectroscopy. Low polar constituents of the specimens were analyzed by the established method. The spectroscopic data were analyzed by chemometric pattern recognition methods
TABLE 1: Information of all the wild Wolfiporia extensa (Peck) Ginns samples.

\begin{tabular}{lc}
\hline Code & Geographical origin \\
\hline C-1 & Chuxiong, Yunnan \\
C-2 & Chuxiong, Yunnan \\
C-3 & Chuxiong, Yunnan \\
C-4 & Chuxiong, Yunnan \\
C-5 & Chuxiong, Yunnan \\
C-6 & Chuxiong, Yunnan \\
C-7 & Chuxiong, Yunnan \\
C-8 & Chuxiong, Yunnan \\
H-1 & Honghe, Yunnan \\
H-2 & Honghe, Yunnan \\
H-3 & Honghe, Yunnan \\
H-4 & Honghe, Yunnan \\
H-5 & Honghe, Yunnan \\
H-6 & Honghe, Yunnan \\
H-7 & Honghe, Yunnan \\
P-1 & Pu'er, Yunnan \\
P-2 & Pu'er, Yunnan \\
P-3 & Pu'er, Yunnan \\
P-4 & Pu'er, Yunnan \\
P-5 & Pu'er, Yunnan \\
P-6 & Pu'er, Yunnan \\
P-7 & Pu'er, Yunnan \\
P-8 & Pu’er, Yunnan \\
\hline
\end{tabular}

such as PLS-DA and HCA. This method could contribute to providing far more information for discriminating medicinal fungi rapidly and accurately.

\section{Materials and Methods}

2.1. Raw Materials. A total of 23 wild W. extensa sclerotium samples were obtained from different regions in Yunnan, southwestern China. Concretely, eight samples of Chuxiong, seven samples from Honghe, and eight samples collected in Pu'er were analyzed in this study. All the samples were authenticated by Dr. Honggao Liu from the College of Food Science and Technology, Yunnan Agricultural University, and preserved in the specimen room of Institute of Medicinal Plants, Yunnan Academy of Agricultural Sciences. The sample information is listed in Table 1.

2.2. Sample Preparation. All the fresh samples were cleaned up and air-dried in the shade after collection. Then they were ground into powder and passed through a 100-mesh stainless steel sieve. The sieved powders were stored in the labeled Ziploc bags at room temperature until further analysis. Each sample $(1.00 \mathrm{~g})$ was dissolved in $10.0 \mathrm{~mL}$ of chloroform (analytical grade), which provided a solvent of low polar constituents of $W$. extensa and extracted by ultrasonication for $40 \mathrm{~min}$. The extracts then were filtered and kept as stock solutions for testing. 
2.3. Ultraviolet Spectroscopy. Each stock solution was analyzed by UV spectroscopy using a TU-1901 PC UV-visible spectrophotometer (Pgeneral, Beijing, China) equipped with a quartz cell with an optical path of $1 \mathrm{~cm}$. The absorption spectra were collected in the working range from 190 to $450 \mathrm{~nm}$ with $0.5 \mathrm{~nm}$ sampling interval and $2.0 \mathrm{~nm}$ slit width. Then the raw spectra were treated by smoothing in order to eliminate the solvent interference and increase accuracy of spectra. The obtained absorption readings of all the samples were converted into a data matrix by using Microsoft Excel 2007 (Microsoft, USA) with the wavelength as variables represented by columns and the corresponding spectral absorbance measurements of different samples represented by rows.

2.4. Data Analysis. In order to sort the W. extensa sclerotium samples according to their geographical origins, the spectrum data matrices were integrated and exported to the appropriate software for chemometrics analysis of the spectra. The chemometric techniques such as multivariate classification methods aimed at finding mathematical models were able to recognize the membership of each sample to its appropriate class, on the basis of a set of measurements [45]. In this study, partial least squares discriminant analysis (PLS-DA) and hierarchical cluster analysis (HCA) were used as multivariate tools. The SIMCA- $\mathrm{P}^{+} 10.0$ (Umetrics, Umeå, Sweden) was used for the PLS-DA modeling, while HCA was carried out using SPSS 20.0 (IBM Corp., Armonk, USA).

According to the algorithm of PLS-DA [46], the spectra of training set can be represented as an $n \times p$ matrix $X$, where $n$ means training objects and $p$ stands for wavelength points. Then an $n \times s$ matrix $Y$ is designed. $s$ is the value of sorted number in this study, $s=3$ (the classes of Chuxiong, Honghe, and Pu'er, resp.). The value of each element in $Y$ is the corresponding class of the object in $X$. If an object $i$ $(i=1: n)$ is from class $j(j=1: s)$, the element at $i$ th row and $j$ th column in $Y$ is given a value of 1 . All other elements in $Y$ are set as -1 . PLS-DA was selected to obtain the first understanding of the relationships among the data matrix and employed to distinguish samples according to their origins. Then, for HCA, the squared Euclidean distance and the average linkage method were used. The main principle of $\mathrm{HCA}$ is assuming that there are $m$ observations; then the algorithm starts with $m$ clusters. With the calculation of the squared Euclidean distance between observations, the closest points are grouped into a single cluster and repeat the process until all the observations are included in one cluster [47]. This method was utilized to evaluate the relationships in terms of similarity or dissimilarity among groups of multivariate data.

\section{Results and Discussion}

3.1. Optimization of Extraction Methods. Six hundred milligrams of every powdered sample was taken out to form the mixed $W$. extensa sclerotium sample. The mixed sample was used to make sure of the optimization of extraction methods. Efficient extraction methods are also required for the highest

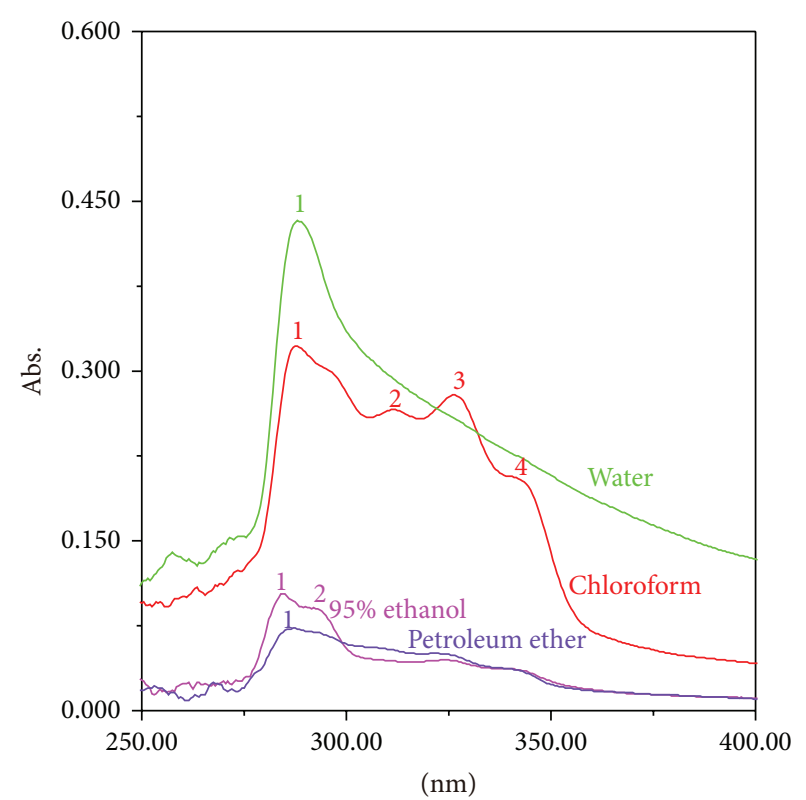

FIGURE 1: UV spectra of different extraction solvent.

extraction efficiency [48]. In order to obtain the efficient extraction methods, the extraction solvent and ultrasonic time tests were investigated. The number of the absorption peaks from four different extracts which were extracted by petroleum ether, chloroform, 95\% ethanol, and ultrapure water was used to validate the extraction solvent while different extraction times (30,40, and $50 \mathrm{~min})$ were screened based on the intensities of absorption bands. All reagents were of analytical grade. The results showed that chloroform could be the most appropriate solvent. Figure 1 shows that the number of the absorption peaks of the chloroform extract is the highest among all the extracts. Others have only one or two absorption peaks. This implied that chloroform extract may obtain more component information about the sample to reflect its characteristic. Moreover, all spectrophotometric signals were maximized with 40 min of extraction and a longer time was not necessary (Figure 2).

3.2. Validation of Methodology. To ensure the validity of this proposed method, the method precision was performed on seven replicate determinations of the extract of mixed sample with the selected condition. The variation of wavelength of common peaks was expressed as relative standard deviation (RSD). The RSDs of precision for this method were less than $1.08 \%$. The repeatability was assessed by testing seven independently prepared extracts which were from the mixed sample using the uniform method. The RSDs of wavelength of common peaks were arranged from 0 to $0.58 \%$. The sample stability was determined by analysing a single sample solution stored at room temperature for $30 \mathrm{~h}$. The RSDs from stability test were below $1.02 \%$ for all the wavelengths of common peaks, indicating that $W$. extensa sclerotium extraction solution was stable within $30 \mathrm{~h}$. These results displayed that this method was reasonable. 


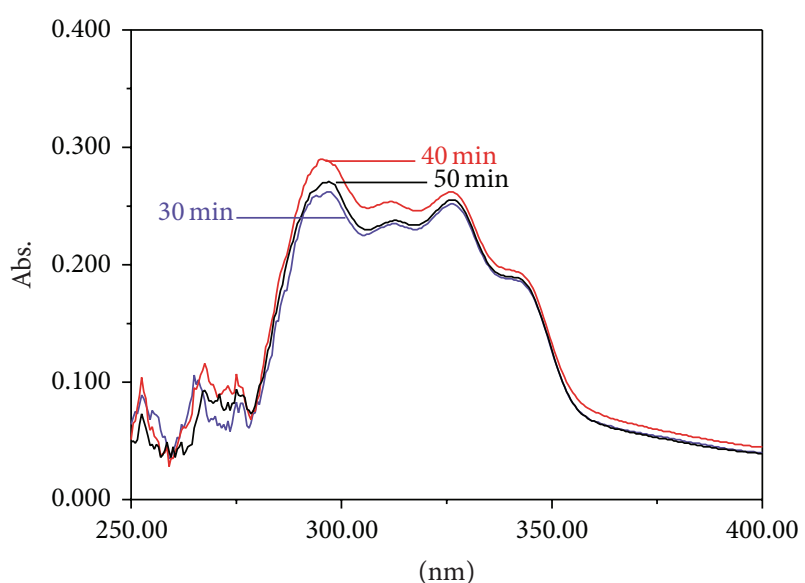

Figure 2: UV spectra of different extraction times.

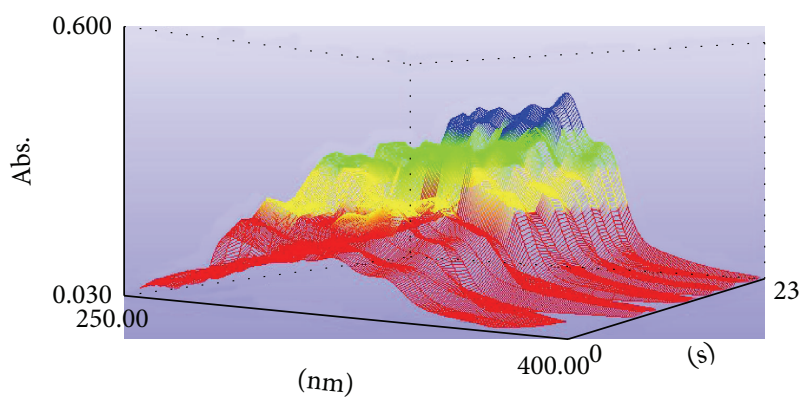

FIGURE 3: Three-dimensional wireframe plot of UV spectra of $W$. extensa sclerotium specimens.

3.3. Spectroscopic Analysis of Low Polar Constituents of W. extensa Sclerotium. The UV absorption bands of the presented samples are usually associated with the presence of different chromophores exemplified in conjugated systems as well as other UV-absorbing systems [49]. The UV spectrum of each of the studied $W$. extensa sclerotium samples was recorded in the region between 190 and $400 \mathrm{~nm}$. On account of the detection range of the UV-visible spectrometer, we chose the wavelengths of absorption peaks arranged from 250 to $400 \mathrm{~nm}$ for the sake of avoiding the spectral noise. The UV spectra for all the samples are presented in Figures 3 and 4.

In the three-dimensional wireframe plot of UV spectra (Figure 3), the red part means the absorbance is recorded in the region between 0 and 0.2 , the yellow one means the absorbance is arranged from 0.2 to 0.3 , for the green part, the absorbance is recorded from 0.3 to 0.4 , and the blue one which means the absorbance is relatively high is arranged from 0.4 to 0.5 . Just a few samples have higher absorbance, which have been shown in blue. It indicated that the absorbance of low polar constituents of different samples has visible differences. To a certain degree, when the substance was in high concentration, the corresponding absorbance was high too [50]. It implied that the contents of low polar constituents of the samples may differ with the geographical origins. For the two-dimensional spectra diagram (Figure 4), the UV spectra fingerprints of low polar

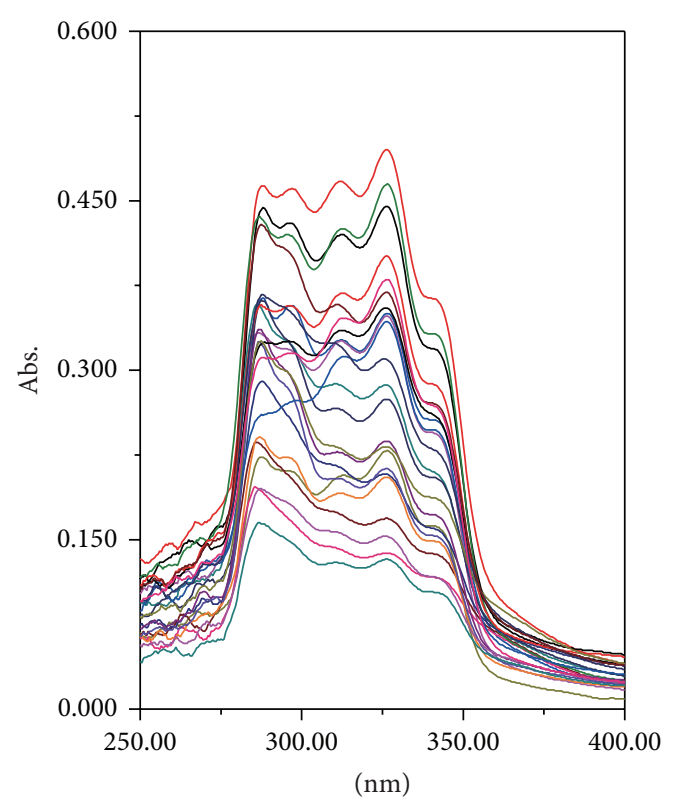

Figure 4: Two-dimensional spectra diagram of $W$. extensa sclerotium samples.

constituents of $W$. extensa sclerotium have high overlap rate from 250 to $400 \mathrm{~nm}$. Every sample has some characteristic absorption peaks to show its fingerprint feature. Some low polar constituents appear to be very similar among these samples because all the samples have some common peaks such as 287, 312, 326, and $340 \mathrm{~nm}$. However, there were obviously differences among the number of absorption peaks and peak positions of these samples. These differences were conducive to discriminate the W. extensa sclerotium samples by showing the fingerprint characteristics.

3.4. Partial Least Squares Discriminant Analysis. PLS-DA, a supervised method, is a variation of PLS analysis. It is considered as a pair comparison analysis and is built to classify a group of samples as belonging or not belonging to a specific class [51, 52]. This method, as a representative technique, was applied to construct and validate a statistical model to find difference in low polar constituents among the $W$. extensa sclerotium samples according to their geographical origins. Figure 5 reports the distance to model in X-space (DModX) of all the samples. The values of DModX of all samples are under 1.45 and a value of $P<0.05$ is considered statistically significant. It revealed that the results of PLS-DA were reasonable.

Figure 6 presents a score plot with 95\% confidence ellipses obtained by applying PLS-DA to the overall set of UV spectra. A separation among the sclerotium samples which were clustered into three classes according to samples collected from Chuxiong, Honghe, and Pu'er, respectively, can be clearly observed in the two-dimensional diagram. As can be seen, it demonstrated the absence of significant variance within the same variety that the samples which were collected from the same origin could get together with each other and be distinct from others. This finding indicated that 


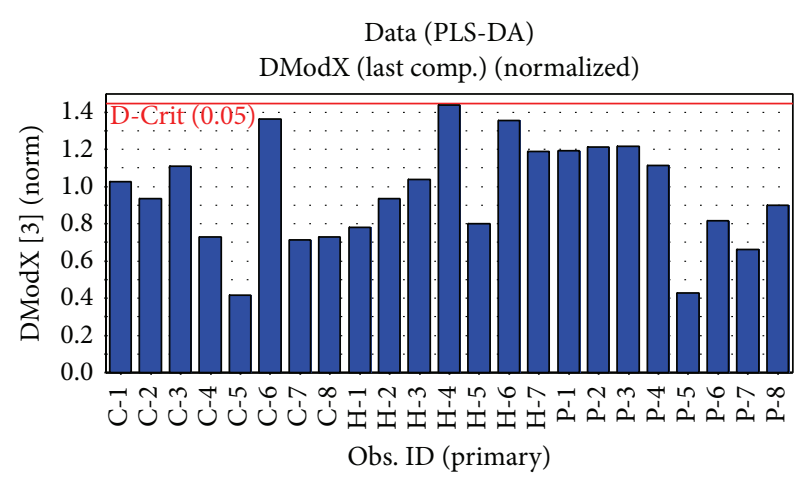

Figure 5: Distance to model in X-space (DModX) of all the specimens.

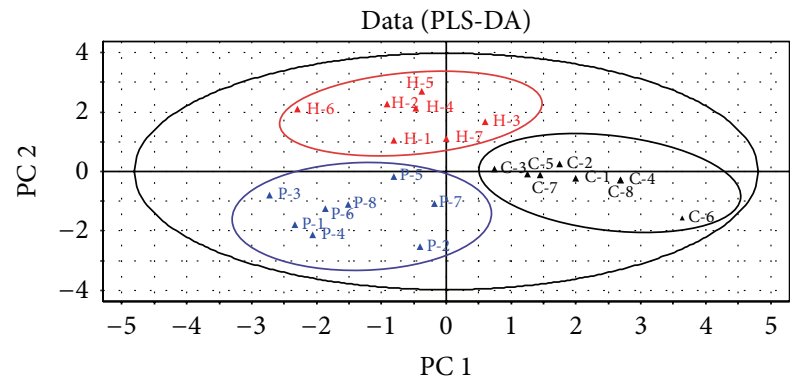

Ellipse: Hotelling T2 (0.95)

- Chuxiong, Yunnan

. Honghe, Yunnan

^ Pu'er, Yunnan

Figure 6: PLS-DA score plot based on UV spectra of W. extensa samples.

the spectral differences among these samples were systematic and can be used for discrimination purposes. Moreover, it could give us a preliminary overview of similarities and diversity among the geographical origins. In addition, all the sample symbols are in the ellipse that proved the effectiveness of the PLS-DA score plot as a convenient visualization technique for the differentiation. As a result, 23 test samples were discriminated entirely as their groups by geographical origins.

According to the spectrophotometric PLS-DA model, a series of scores (variable importance for the projections (VIPs)) were computed to express the contribution of absorbance to these dimensions. A variable is usually considered important to the model when its VIP is above 1.0 [53]. Figure 7 and Table 2 have shown the VIP scores of the PLS-DA. The samples' data are easily discernible. Components that play important roles in the separation are picked out according to the parameter VIP. As shown in this figure, the absorption of the wavelength of $326.5,287.5,287$, $262.5,285.5,326,252.5,255.5,288,311,254.5$, and $312 \mathrm{~nm}$ is likely to be considered as main factor for discrimination of all these specimens. From the corresponding loading plot (Figure 8), PC 1 resolves the measured composition profiles of specimens collected from Chuxiong from other samples.

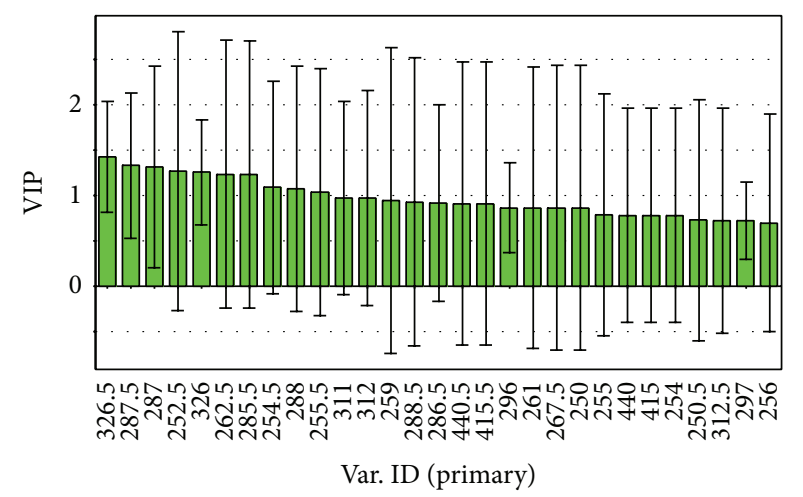

FIGURE 7: Variable importance for the projection (VIP) plot of absorbance for the contribution to sample separation from PLS-DA.

TABLE 2: VIP scores of PLS-DA.

\begin{tabular}{|c|c|}
\hline Var. ID (primary) & VIP \\
\hline 326.5 & 1.51178 \\
\hline 287.5 & 1.40302 \\
\hline 287 & 1.38853 \\
\hline 262.5 & 1.30931 \\
\hline 285.5 & 1.30725 \\
\hline 326 & 1.27077 \\
\hline 252.5 & 1.25123 \\
\hline 255.5 & 1.10071 \\
\hline 288 & 1.09983 \\
\hline 311 & 1.0315 \\
\hline 254.5 & 1.01802 \\
\hline 312 & 1.00679 \\
\hline 286.5 & 0.971985 \\
\hline 288.5 & 0.953564 \\
\hline 267.5 & 0.901482 \\
\hline 250 & 0.901482 \\
\hline 259 & 0.88801 \\
\hline 440.5 & 0.810888 \\
\hline 415.5 & 0.810888 \\
\hline 250.5 & 0.773825 \\
\hline 312.5 & 0.767684 \\
\hline 255 & 0.759275 \\
\hline 440 & 0.720724 \\
\hline 415 & 0.720724 \\
\hline 254 & 0.720724 \\
\hline 256 & 0.707384 \\
\hline 261 & 0.699291 \\
\hline 297 & 0.627387 \\
\hline 296 & 0.62471 \\
\hline
\end{tabular}

This variation was mainly attributable to the absorbance of wavelength of 326,311 , and $254.5 \mathrm{~nm}$, of which the loading values were positive. In addition, the samples of Honghe were separated from those of Pu'er by PC 2 in which the corresponding loading values were positive for the absorbance of wavelength of $312,285.5,262.5$, and $252.5 \mathrm{~nm}$. What is more, 
TABle 3: Agglomeration schedule of HCA.

\begin{tabular}{|c|c|c|c|c|c|c|}
\hline \multirow{2}{*}{ Stage } & \multicolumn{2}{|c|}{ Cluster combination } & \multirow{2}{*}{ Coefficients } & \multicolumn{2}{|c|}{ Stage cluster first appearance } & \multirow{2}{*}{ Next stage } \\
\hline & Cluster 1 & Cluster 2 & & Cluster 1 & Cluster 2 & \\
\hline 1 & 4 & 8 & 0.000 & 0 & 0 & 15 \\
\hline 2 & 5 & 7 & 0.040 & 0 & 0 & 6 \\
\hline 3 & 21 & 23 & 0.142 & 0 & 0 & 11 \\
\hline 4 & 16 & 19 & 0.187 & 0 & 0 & 11 \\
\hline 5 & 10 & 12 & 0.216 & 0 & 0 & 8 \\
\hline 6 & 2 & 5 & 0.292 & 0 & 2 & 7 \\
\hline 7 & 1 & 2 & 0.393 & 0 & 6 & 10 \\
\hline 8 & 10 & 13 & 0.395 & 5 & 0 & 16 \\
\hline 9 & 11 & 15 & 0.659 & 0 & 0 & 14 \\
\hline 10 & 1 & 3 & 0.881 & 7 & 0 & 15 \\
\hline 11 & 16 & 21 & 0.947 & 4 & 3 & 13 \\
\hline 12 & 20 & 22 & 1.196 & 0 & 0 & 17 \\
\hline 13 & 16 & 18 & 1.457 & 11 & 0 & 17 \\
\hline 14 & 9 & 11 & 1.526 & 0 & 9 & 16 \\
\hline 15 & 1 & 4 & 1.834 & 10 & 1 & 20 \\
\hline 16 & 9 & 10 & 1.984 & 14 & 8 & 19 \\
\hline 17 & 16 & 20 & 3.922 & 13 & 12 & 18 \\
\hline 18 & 16 & 17 & 4.365 & 17 & 0 & 21 \\
\hline 19 & 9 & 14 & 4.569 & 16 & 0 & 21 \\
\hline 20 & 1 & 6 & 6.022 & 15 & 0 & 22 \\
\hline 21 & 9 & 16 & 13.409 & 19 & 18 & 22 \\
\hline 22 & 1 & 9 & 14.743 & 20 & 21 & 0 \\
\hline
\end{tabular}

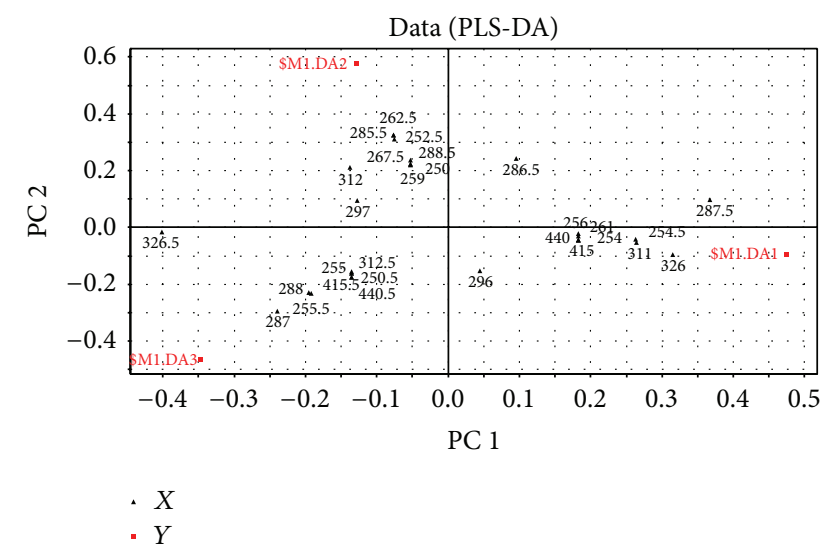

FIgURE 8: Loading plot generated from the PLS-DA model of the $W$. extensa samples.

these methods could screen suitable wavelengths to provide references for the quantitative test. Combining the spectra diagram with the VIP scores, the wavelengths of 287 and $326 \mathrm{~nm}$ may be appropriate for quantitative test because they were the common peaks of the samples and had the VIPs greater than 1.0 as well.

3.5. Hierarchical Cluster Analysis. HCA is an unsupervised pattern recognition method for clustering samples based on their similarities $[54,55]$. To further explore the relationships among the $W$. extensa sclerotium specimens, HCA of the spectra data was performed. Table 3 is the agglomeration schedule that shows the detailed steps of HCA. The corresponding cluster dendrogram was generated by applying hclust function using average linkage clustering of the squared Euclidean distance based on the normalized data from the 23 test samples. As shown in Figure 9, all the specimens could be divided into three fractions when the distance of them is twenty, group I contains the samples of Chuxiong, and the other two groups are composed of the samples collected from Pu'er and Honghe, respectively. All samples were correctly classified according to their geographical origins without any misclassification. In addition, the results could verify the consequence of PLS-DA.

It suggested that the low polar constituents in W. extensa sclerotium collected from the same area may be similar while the accumulation of these chemical constituents in samples that had different collection sites was likely to be diverse. This may be related to the local environment factors such as temperature, rainfall, soil type, vegetation type, or other characteristics. Similar results have been reported in the previous researches that phytochemical composition and metabolites of medicinal fungi could be affected by the geographical origins $[9,56]$. This study implied that samples collected from different sites could be clearly distinguished by UV characteristic fingerprint based on low polar constituents when combined with chemometrics. Compared with the similar study that uses UV spectra fingerprint in combination with the common and variation peak ratio dual index 


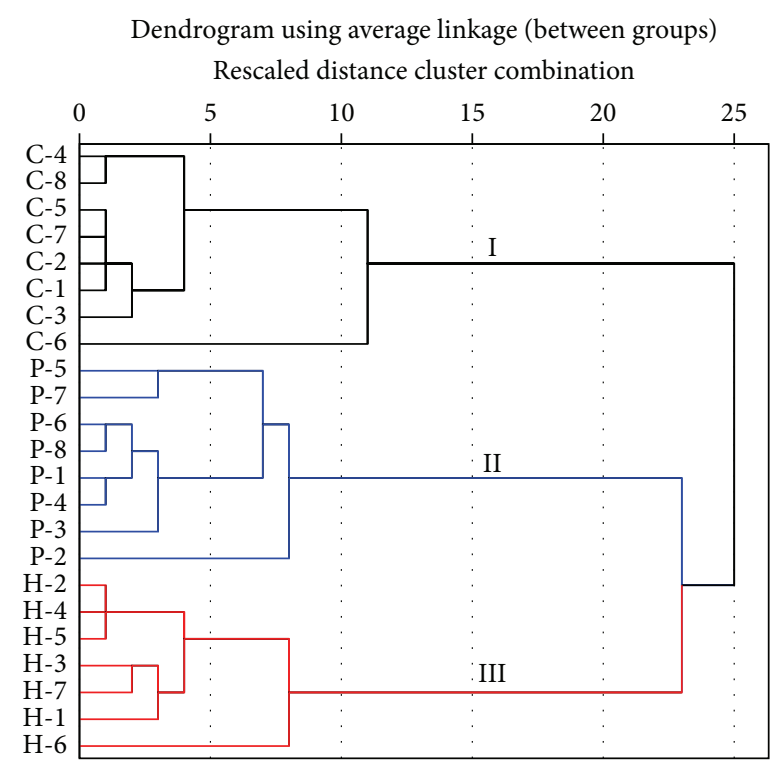

FIGURE 9: Dendrogram resulting from hierarchical cluster analysis.

sequence analysis for qualitative evaluation and reveals the differences of specimens collected from different areas [57], our study has the advantageous aspects that the results were visualized for the differentiation of samples and it was a convenient approach avoiding the tedious calculation. In addition, in contrast to the results of previous studies related to the discrimination of medicinal fungi based on other analytical approaches, such as HPLC [17] and GC-MS [58], it can be concluded that the proposed method is a reliable and fast tool for discriminating medicinal fungi.

\section{Conclusions}

In conclusion, a novel, fast, and convenient method has been developed to differentiate the sclerotium of $W$. extensa from different geographical sites based on low polar constituents by using UV spectroscopy coupled with chemometrics methods. All the samples could be discriminated accurately according to their origins. Even though the proposed method is qualitative, it avoids the need of a quantitative method that would require the use of standards, calibration, and timeconsuming analysis. Furthermore, this approach is simple, of low cost, and reliable and has a significant advantage for discrimination of other species of medicinal fungi.

\section{Conflict of Interests}

The authors declare that there is no conflict of interests regarding the publication of this paper.

\section{Acknowledgments}

This work was sponsored by the National Natural Science Foundation of China (Grant no. 31460538). The authors are grateful for the financial support.

\section{References}

[1] O. V. Oyetayo, "Medicinal uses of mushrooms in Nigeria: towards full and sustainable exploitation," African Journal of Traditional, Complementary and Alternative Medicines, vol. 8, no. 3, pp. 267-274, 2011.

[2] S. Wasser, "Medicinal mushrooms as a source of antitumor and immunomodulating polysaccharides," Applied Microbiology and Biotechnology, vol. 60, no. 3, pp. 258-274, 2003.

[3] C. Lull, H. J. Wichers, and H. F. J. Savelkoul, "Antiinflammatory and immunomodulating properties of fungal metabolites," Mediators of Inflammation, vol. 2005, no. 2, pp. 63-80, 2005.

[4] State Pharmacopoeia Commission, Chinese Pharmacopoeia, Chemistry and Industry Press, Beijing, China, 2010.

[5] C. W. Phan, P. David, M. Naidu, K. H. Wong, and V. Sabaratnam, "Therapeutic potential of culinary-medicinal mushrooms for the management of neurodegenerative diseases: diversity, metabolite, and mechanism," Critical Reviews in Biotechnology. In press.

[6] Y.-K. Choong, C.-H. Xu, J. Lan, X.-D. Chen, and J. A. Jamal, "Identification of geographical origin of Lignosus samples using Fourier transform infrared and two-dimensional infrared correlation spectroscopy," Journal of Molecular Structure, vol. 1069, no. 1, pp. 188-195, 2014.

[7] Y. Chen, Y. Yan, M.-Y. Xie et al., "Development of a chromatographic fingerprint for the chloroform extracts of Ganoderma lucidum by HPLC and LC-MS," Journal of Pharmaceutical and Biomedical Analysis, vol. 47, no. 3, pp. 469-477, 2008.

[8] A. K. Panda and K. C. Swain, "Traditional uses and medicinal potential of Cordyceps sinensis of Sikkim," Journal of Ayurveda and Integrative Medicine, vol. 2, no. 1, pp. 9-13, 2011.

[9] L.-X. Guo, X.-M. Xu, C.-F. Wu et al., "Fatty acid composition of lipids in wild Cordyceps sinensis from major habitats in China," Biomedicine \& Preventive Nutrition, vol. 2, no. 1, pp. 42-50, 2012.

[10] Y. Zhang, F. Li, F. Huang et al., "Metabolomics analysis reveals variation in Schisandra chinensis cetabolites from different origins," Journal of Separation Science, vol. 37, no. 6, pp. 731-737, 2014.

[11] World Health Organization, Guidelines for the Assessment of Herbal Medicines, World Health Organization, Geneva, Switzerland, 1991.

[12] Technical Requirements for the Development of Finger-prints of TCM Injections, State Food and Drug Administration of China, Beijing, China, 2000.

[13] H. Sheridan, L. Krenn, R. Jiang et al., “The potential of metabolic fingerprinting as a tool for the modernisation of TCM preparations," Journal of Ethnopharmacology, vol. 140, no. 3, pp. 482491, 2012.

[14] D.-Z. Yang, Y.-Q. An, X.-L. Jiang et al., "Development of a novel method combining HPLC fingerprint and multi-ingredients quantitative analysis for quality evaluation of traditional Chinese medicine preparation," Talanta, vol. 85, no. 2, pp. 885-890, 2011.

[15] N. Xu, W.-Q. Luo, and H.-Q. Yang, "Rapid discrimination of fermented Cordyceps mycelium powder by near-infrared spectroscopy (NIRS)," Applied Mechanics and Materials, vol. 302, pp. 189-193, 2013.

[16] M. Mularczyk-Oliwa, A. Bombalska, M. Kaliszewski et al., "Rapid discrimination of several fungus species with FTIR spectroscopy and statistical analysis," Biuletyn Wojskowej Akademii Technicznej, vol. 62, no. 3, pp. 71-80, 2013. 
[17] Y. Chen, S.-B. Zhu, M.-Y. Xie et al., "Quality control and original discrimination of Ganoderma lucidum based on highperformance liquid chromatographic fingerprints and combined chemometrics methods," Analytica Chimica Acta, vol. 623, no. 2, pp. 146-156, 2008.

[18] Y. Chen, W. Bicker, J. Wu, M. Xie, and W. Lindner, "Simultaneous determination of 16 nucleosides and nucleobases by hydrophilic interaction chromatography and its application to the quality evaluation of Ganoderma," Journal of Agricultural and Food Chemistry, vol. 60, no. 17, pp. 4243-4252, 2012.

[19] R. Malheiro, P. G. de Pinho, S. Soares, A. C. da Silva Ferreira, and P. Baptista, "Volatile biomarkers for wild mushrooms species discrimination," Food Research International, vol. 54, no. 1, pp. 186-194, 2013.

[20] E. A. Abella, G. D. Robson, and R. F. Preziosi, "Molecular discrimination of wild Philippine paddy straw mushroom (Volvariella volvacea)," Journal of Life Sciences, vol. 8, no. 1, pp. 27-34, 2014.

[21] M. Shen, M. Xie, S. Nie, Y. Wan, and J. Xie, "Discrimination of different Ganoderma species and their region based on GCMS profiles of sterols and pattern recognition techniques," Analytical Letters, vol. 44, no. 5, pp. 863-873, 2011.

[22] Y. Chen, M.-Y. Xie, Y. Yan et al., "Discrimination of Ganoderma lucidum according to geographical origin with near infrared diffuse reflectance spectroscopy and pattern recognition techniques," Analytica Chimica Acta, vol. 618, no. 2, pp. 121-130, 2008.

[23] P. Geladi, "Chemometrics in spectroscopy. Part 1. Classical chemometrics," Spectrochimica Acta B, vol. 58, no. 5, pp. 767-782, 2003.

[24] R. G. Brereton, "A short history of chemometrics: a personal view," Journal of Chemometrics, vol. 28, no. 10, pp. 749-760, 2014.

[25] D. J. Wang, X. Y. Zhou, T. M. Jin, X. N. Hu, J. E. Zhong, and Q. $\mathrm{T}$. Wu, "Application of near-infrared spectroscopy to agriculture and food analysis," Spectroscopy and Spectral Analysis, vol. 24, no. 4, pp. 447-450, 2004.

[26] A. Fernández-González, J. M. Montejo-Bernardo, H. Rodríguez- Prieto, C. Castaño-Monllor, R. Badía-Laíño, and M. E. Díaz-García, "Easy-to-use analytical approach based on ATR-FTIR and chemometrics to identify apple varieties under Protected Designation of Origin (PDO)," Computers and Electronics in Agriculture, vol. 108, pp. 166-172, 2014.

[27] A.-R. A. Roshan, H. A. Gad, S. H. El-Ahmady, M. S. Khanbash, M. I. Abou-Shoer, and M. M. Al-Azizi, "Authentication of monofloral yemeni sidr honey using ultraviolet spectroscopy and chemometric analysis," Journal of Agricultural and Food Chemistry, vol. 61, no. 32, pp. 7722-7729, 2013.

[28] H. A. Gad, S. H. El-Ahmady, M. I. Abou-Shoer, and M. M. AlAzizi, "A modern approach to the authentication and quality assessment of thyme using UV spectroscopy and chemometric analysis," Phytochemical Analysis, vol. 24, no. 6, pp. 520-526, 2013.

[29] T. W. Yang, B. K. Cui, J. Zhang et al., "Identification of different parts of edible bolete mushrooms by UV fingerprint," Mycosyst, vol. 33, no. 2, pp. 262-272, 2014.

[30] T. W. Yang, T. Li, J. Zhang, J. Q. Li, H. G. Liu, and Y. Z. Wang, "Rapid identification of bolete mushroom by UV spectroscopy combined with Euclidean distance and principal component analysis," Food Science, vol. 35, no. 16, pp. 105-109, 2014.

[31] X. L. Wu, X. L. Mao, G. E. Tuli et al., Medicinal Fungi of China, Science Press, Beijing, China, 1st edition, 2013.
[32] Y. Ling, M. C. Chen, K. Wang et al., "Systematic screening and characterization of the major bioactive components of Poria cocos and their metabolites in rats by LC-ESI-MS ${ }^{n}$," Biomedical Chromatography, vol. 26, no. 9, pp. 1109-1117, 2012.

[33] J. L. Ríos, "Chemical constituents and pharmacological properties of Poria cocos," Planta Medica, vol. 77, no. 7, pp. 681-691, 2011.

[34] J.-B. Chen, S.-Q. Sun, F. Ma, and Q. Zhou, "Vibrational microspectroscopic identification of powdered traditional medicines: chemical micromorphology of Poria observed by infrared and Raman microspectroscopy," Spectrochimica Acta Part A: Molecular and Biomolecular Spectroscopy, vol. 128, no. 15, pp. 629-637, 2014.

[35] Y.-Z. Wang, J. Zhang, Y.-L. Zhao et al., "Mycology, cultivation, traditional uses, phytochemistry and pharmacology of Wolfporia cocos (Schwein.) Ryvarden et Gilb.: a review," Journal of Ethnopharmacology, vol. 147, no. 2, pp. 265-276, 2013.

[36] J. Tang, J. Nie, D. Li et al., "Characterization and antioxidant activities of degraded polysaccharides from Poria cocos sclerotium," Carbohydrate Polymers, vol. 105, no. 25, pp. 121-126, 2014.

[37] L. Zhou, Y. Zhang, L. A. Gapter, H. Ling, R. Agarwal, and K.Y. Ng, "Cytotoxic and anti-oxidant activities of lanostane-type triterpenes isolated from Poria cocos," Chemical \& Pharmaceutical Bulletin, vol. 56, no. 10, pp. 1459-1462, 2008.

[38] L. Gapter, Z. Wang, J. Glinski, and K.-Y. Ng, "Induction of apoptosis in prostate cancer cells by pachymic acid from Poria cocos," Biochemical and Biophysical Research Communications, vol. 332, no. 4, pp. 1153-1161, 2005.

[39] T. G. Cai and Y. Cai, "Triterpenes from the fungus Poria cocos and their inhibitory activity on nitric oxide production in mouse macrophages via blockade of activating protein-1 pathway," Chemistry and Biodiversity, vol. 8, no. 11, pp. 21352143, 2011.

[40] G. H. Li, Y. M. Shen, and K. Q. Zhang, "Nematicidal activity and chemical component of Poria cocos," The Journal of Microbiology, vol. 43, no. 1, pp. 17-20, 2005.

[41] S. M. Lee, Y. J. Lee, J. J. Yoon, D. G. Kang, and H. S. Lee, "Effect of Poria cocos on hypertonic stress-induced water channel expression and apoptosis in renal collecting duct cells," Journal of Ethnopharmacology, vol. 141, no. 1, pp. 368-376, 2012.

[42] C. L.-T. Chang, T.-H. Li, C.-C. Hou, and W.-C. Yang, "Antihyperglycemic properties of crude extract and triterpenes from Poria cocos," Evidence-based Complementary and Alternative Medicine, vol. 2011, Article ID 128402, 8 pages, 2011.

[43] M. K. Lu, J. J. Cheng, C. Y. Lin, and C. C. Chang, "Purification, structural elucidation, and anti-inflammatory effect of a watersoluble 1,6-branched 1,3- $\alpha$-d-galactan from cultured mycelia of Poria cocos," Food Chemistry, vol. 118, no. 2, pp. 349-356, 2010.

[44] X. Chen, Q. Tang, Y. Chen, W. Wang, and S. Li, "Simultaneous extraction of polysaccharides from Poria cocos by ultrasonic technique and its inhibitory activities against oxidative injury in rats with cervical cancer," Carbohydrate Polymers, vol. 79, no. 2, pp. 409-413, 2010.

[45] D. Ballabio and V. Consonni, "Classification tools in chemistry. Part 1: linear models. PLS-DA," Analytical Methods, vol. 5, no. 16, pp. 3790-3798, 2013.

[46] S. M. Yan, J. P. Liu, L. Xu et al., "Rapid discrimination of the geographical origins of an Oolong tea (Anxi-Tieguanyin) by near-infrared spectroscopy and partial least squares discriminant analysis," Journal of Analytical Methods in Chemistry, vol. 2014, Article ID 704971, 6 pages, 2014. 
[47] J. Z. Ren, S. Tan, and L. Dong, “The assessment of hydrogen energy systems for fuel cell vehicles using principal component analysis and cluster analysis," International Scholarly Research Notices, vol. 2012, Article ID 191308, 8 pages, 2012.

[48] S. Yudthavorasit, K. Wongravee, and N. Leepipatpiboon, "Characteristic fingerprint based on gingerol derivative analysis for discrimination of ginger (Zingiber officinale) according to geographical origin using HPLC-DAD combined with chemometrics," Food Chemistry, vol. 158, no. 1, pp. 101-111, 2014.

[49] Y. E. Zeng and L. Zhang, Instrumental Analysis, Science Press, Beijing, China, 5th edition, 2010.

[50] K.-L. Wei, Z.-Y. Wen, X. Wu, Z.-W. Zhang, and T.-L. Zeng, "Research advances in water quality monitoring technology based on UV-Vis spectrum analysis," Spectroscopy and Spectral Analysis, vol. 31, no. 4, pp. 1074-1077, 2011.

[51] J. H. da Silva Taveira, F. M. Borém, L. P. Figueiredo et al., "Potential markers of coffee genotypes grown in different Brazilian regions: a metabolomics approach," Food Research International, vol. 61, pp. 75-82, 2014.

[52] R. Díaz, O. J. Pozo, J. V. Sancho, and F. Hernández, "Metabolomic approaches for orange origin discrimination by ultra-high performance liquid chromatography coupled to quadrupole time-of-flight mass spectrometry," Food Chemistry, vol. 157, no. 15, pp. 84-93, 2014.

[53] Y.-R. Kang, Y. S. Park, Y. C. Park et al., "UPLC/Q-TOF MS based metabolomics approach to post-mortem-interval discrimination: Mass spectrometry based metabolomics approach," Journal of Pharmaceutical Investigation, vol. 42, no. 1, pp. 41-46, 2012.

[54] C. Luo, T. He, and Z. Chun, "Discrimination and chemical phylogenetic study of seven species of Dendrobium using infrared spectroscopy combined with cluster analysis," Journal of Molecular Structure, vol. 1037, no. 10, pp. 40-48, 2013.

[55] C. Sârbu, R. D. Nacu-Briciu, A. Kot-Wasik, S. Gorinstein, and J. Namieśnik, "Classification and fingerprinting of kiwi and pomelo fruits by multivariate analysis of chromatographic and spectroscopic data," Food Chemistry, vol. 130, no. 4, pp. 994$1002,2012$.

[56] H. G. Liu, T. Li, Y. L. Zhao, J. Zhang, and Y. Z. Wang, "Determination of some metabolites of Cordyceps sobolifera," African Journal of Microbiology Research, vol. 5, no. 30, pp. 5518-5522, 2011.

[57] T. J. Yuan, Y. Z. Wang, Y. L. Zhao, J. Zhang, H. Jin, and J. Y. Zhang, "The common and variation peak ratio dual index sequence analysis in UV fingerprint spectra of Gentiana Rigescens," Spectroscopy and Spectral Analysis, vol. 31, no. 8, pp. 2161-2165, 2011.

[58] I. Marekov, S. Momchilova, B. Grung, and B. NikolovaDamyanova, "Fatty acid composition of wild mushroom species of order Agaricales-examination by gas chromatography-mass spectrometry and chemometrics," Journal of Chromatography B, vol. 910, pp. 54-60, 2012. 

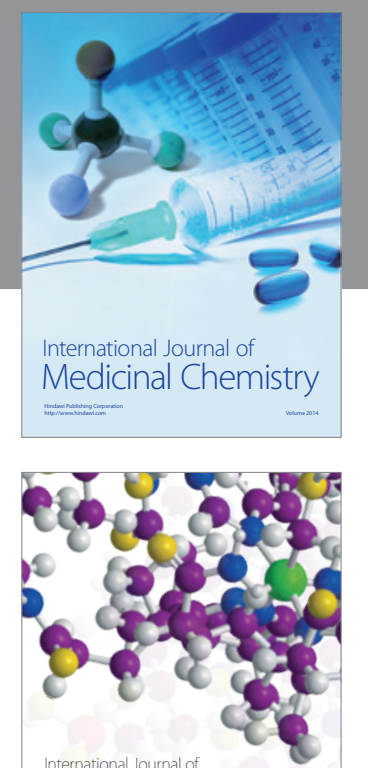

\section{Carbohydrate} Chemistry

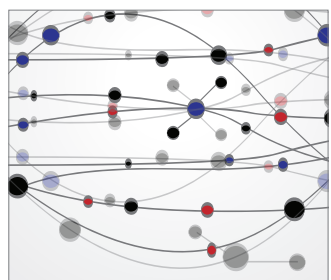

The Scientific World Journal
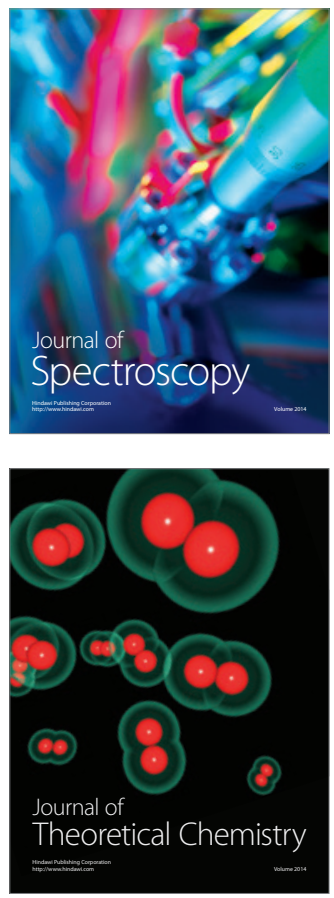
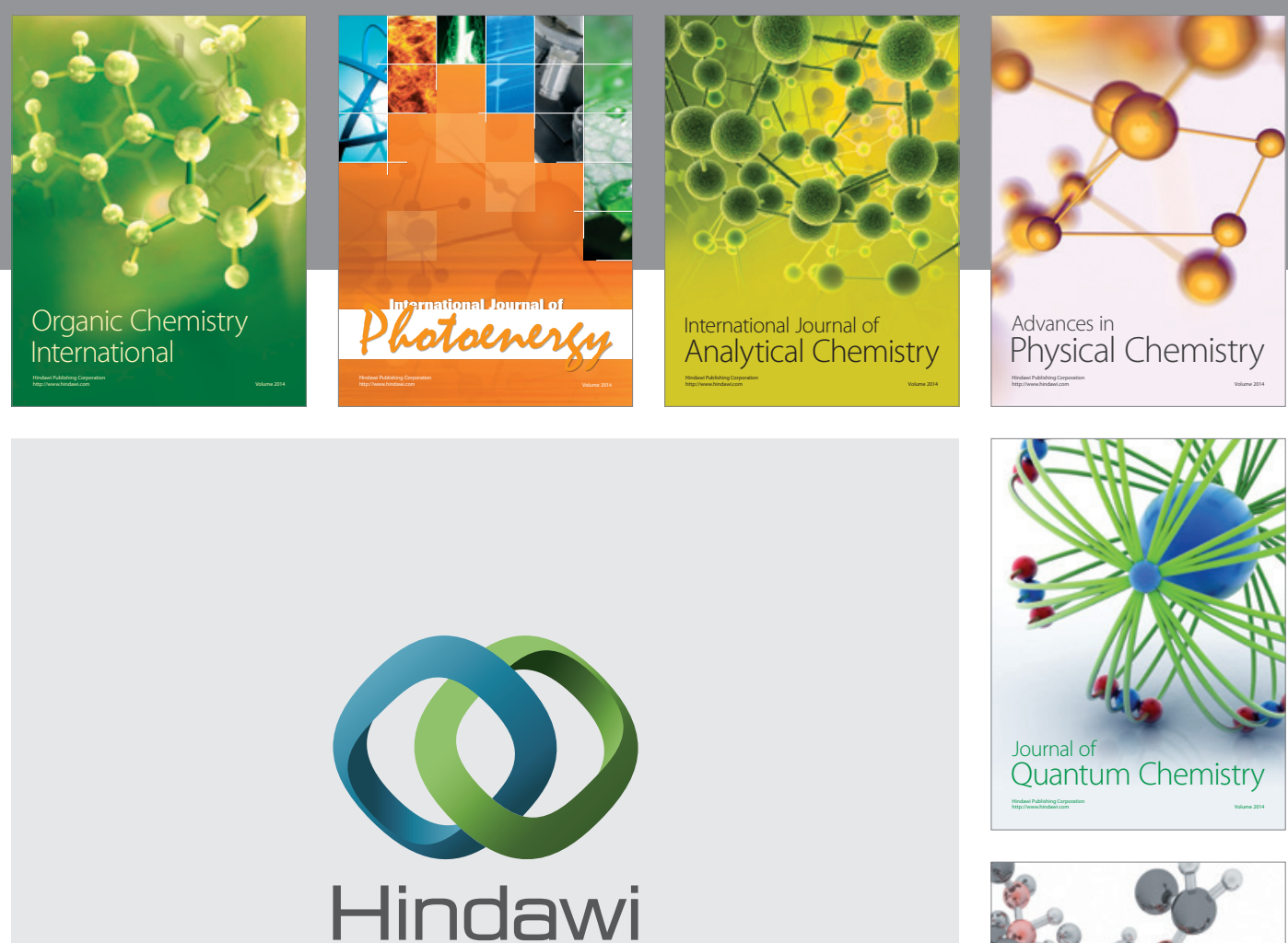

Submit your manuscripts at

http://www.hindawi.com

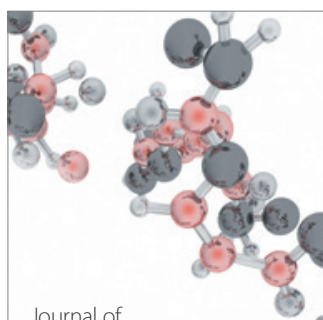

Analytical Methods

in Chemistry

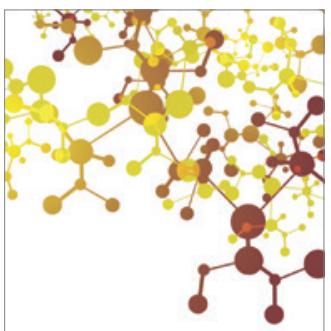

Journal of

Applied Chemistry

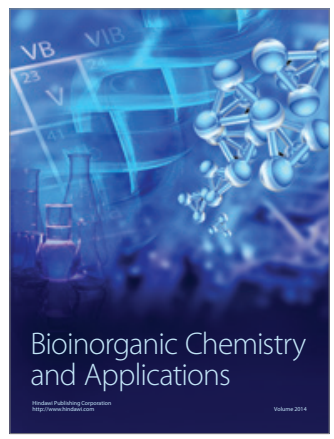

Inorganic Chemistry
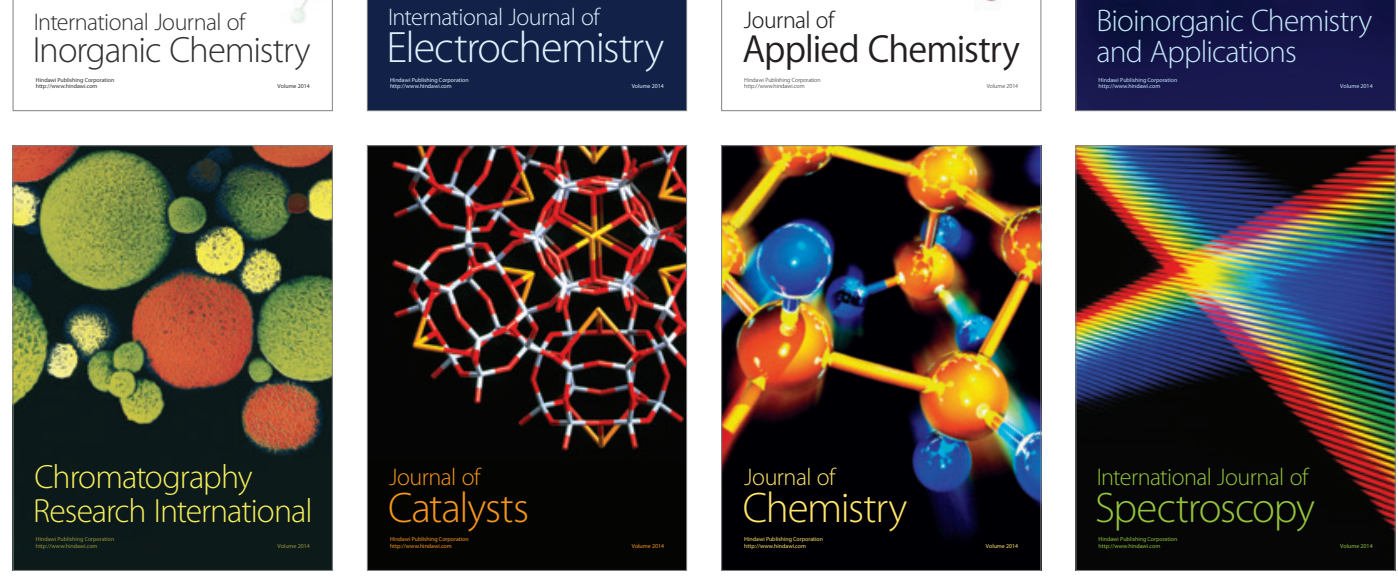